\title{
Electron refraction at lateral atomic interfaces
}

Z. M. Abd El-Fattah, M. A. Kher-Elden, O. Yassin, M. M. El-Okr, J. E. Ortega, and F. J. García de Abajo

Citation: Journal of Applied Physics 122, 195306 (2017); doi: 10.1063/1.5005062

View online: https://doi.org/10.1063/1.5005062

View Table of Contents: http://aip.scitation.org/toc/jap/122/19

Published by the American Institute of Physics

\section{Articles you may be interested in}

Electrostatics of lateral $p-n$ junctions in atomically thin materials

Journal of Applied Physics 122, 194501 (2017); 10.1063/1.4994047

Extracting dielectric fixed charge density on highly doped crystalline-silicon surfaces using photoconductance measurements

Journal of Applied Physics 122, 195301 (2017); 10.1063/1.5010160

Modulation of the electromagnetic local density of states in graphene-based hyperbolic metamaterials Journal of Applied Physics 122, 193101 (2017); 10.1063/1.5003771

Plasmonic metamaterial based unified broadband absorber/near infrared emitter for thermophotovoltaic system based on hexagonally packed tungsten doughnuts

Journal of Applied Physics 122, 193104 (2017); 10.1063/1.5003054

Removing the current-limit of vertical organic field effect transistors Journal of Applied Physics 122, 195502 (2017); 10.1063/1.5005800

Hybrid functional study of native point defects and impurities in $\mathrm{ZnGeN}_{2}$ Journal of Applied Physics 122, 195701 (2017); 10.1063/1.4999790

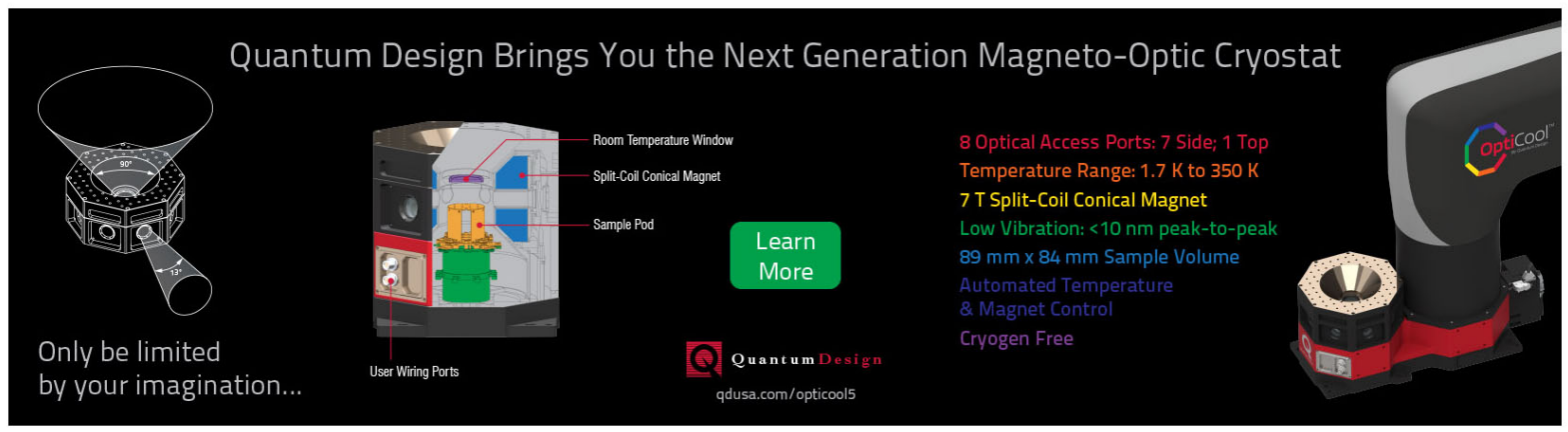




\title{
Electron refraction at lateral atomic interfaces
}

\author{
Z. M. Abd El-Fattah, ${ }^{1,2, a)}$ M. A. Kher-Elden, ${ }^{1}$ O. Yassin, ${ }^{1}$ M. M. El-Okr, ${ }^{1}$ J. E. Ortega,,${ }^{3,4,5}$ \\ and F. J. García de Abajo ${ }^{2,6}$ \\ ${ }^{1}$ Physics Department, Faculty of Science, Al-Azhar University, Nasr City, E-11884 Cairo, Egypt \\ ${ }^{2}$ ICFO-Institut de Ciencies Fotoniques, The Barcelona Institute of Science and Technology, 08860 Castelldefels, \\ Barcelona, Spain \\ ${ }^{3}$ Donostia International Physics Center, Paseo Manuel Lardizábal 4, E-20018 Donostia-San Sebastián, Spain \\ ${ }^{4}$ Centro de Física de Materiales (CSIC-UPV-EHU) and Materials Physics Center (MPC), 20018 San \\ Sebastián, Spain \\ ${ }^{5}$ Departamento de Física Aplicada I, Universidad del País Vasco, 20018 San Sebastián, Spain \\ ${ }^{6}$ ICREA-Institució Catalana de Recerca i Estudis Avançats, Passeig Lluís Companys 23, 08010 Barcelona, Spain
}

(Received 16 September 2017; accepted 31 October 2017; published online 21 November 2017)

\begin{abstract}
We present theoretical simulations of electron refraction at the lateral atomic interface between a "homogeneous" $\mathrm{Cu}(111)$ surface and the "nanostructured" one-monolayer (ML) $\mathrm{Ag} / \mathrm{Cu}(111)$ dislocation lattice. Calculations are performed for electron binding energies barely below the $1 \mathrm{ML} \mathrm{Ag}$ / $\mathrm{Cu}(111) \bar{M}$-point gap (binding energy $E_{B}=53 \mathrm{meV}$, below the Fermi level) and slightly above its $\bar{\Gamma}$-point energy $\left(E_{B}=160 \mathrm{meV}\right)$, both characterized by isotropic/circular constant energy surfaces. Using plane-wave-expansion and boundary-element methods, we show that electron refraction occurs at the interface, the Snell law is obeyed, and a total internal reflection occurs beyond the critical angle. Additionally, a weak negative refraction is observed for $E_{B}=53 \mathrm{meV}$ electron energy at beam incidence higher than the critical angle. Such an interesting observation stems from the interface phase-matching and momentum conservation with the umklapp bands at the second Brillouin zone of the dislocation lattice. The present analysis is not restricted to our $\mathrm{Cu}-\mathrm{Ag} / \mathrm{Cu}$ model system but can be readily extended to technologically relevant interfaces with spinpolarized, highly featured, and anisotropic constant energy contours, such as those characteristic for Rashba systems and topological insulators. Published by AIP Publishing.

https://doi.org/10.1063/1.5005062
\end{abstract}

\section{INTRODUCTION}

Wave-particle duality firmly established for light in the beginning of the twentieth century and later for electrons has set a solid analogue between electrons and photons and brought together much of the understanding of their physics. ${ }^{1}$ For example, band theory first formulated for electrons in crystalline solids has been successfully applied to electromagnetic waves, allowing the extensive study of a variety of artificial periodic one, two, and three-dimensional photonic crystals. ${ }^{2}$ For photonics-based applications, structures with periodicity of the order of the light wavelength $(300-1000 \mathrm{~nm})$ are required, for which lithographic techniques are at hand. ${ }^{3}$ Nanostructured noble metal surfaces that host Shockley-type electronic surface states could be considered electron analogues of photonic systems. However, they are characterized by a Fermi wavelength of the order of $1-3 \mathrm{~nm},{ }^{4}$ so that much finer nanostructuring is required to produce Surface State Nanoelectronics (SSNE). Techniques such as self-assembly and Scanning Tunneling Microscopy (STM) make the fabrication of these nanostructures possible. ${ }^{5,6}$

A case study in the present article is a two-dimensional (2D) lateral superlattice made by combination of two noble metal surfaces with a large lattice mismatch. Such a combination commonly organizes forming moiré superstructures. In

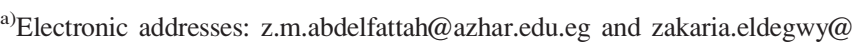
icfo.eu
}

particular, the one monolayer (ML) $\mathrm{Ag} / \mathrm{Cu}(111)$ surface exhibits an irreversible transformation from such a moiré pattern into a hexagonal lattice of dislocations (periodicity $\sim 2.4 \mathrm{~nm}) .^{7,8}$ The peculiar band structure of this system, probed with photoemission and modeled through a numerical plane-wave-expansion, allowed us to conceptually demonstrate electron guiding and collimation of Shockley electrons. Specifically, the self-collimation was reported in the unoccupied states, and therefore, its experimental realization demands sophisticated techniques such as multi-tip STM for simultaneous electron injection and scanning. 9 A striking electron-wave analogue of electron refraction (and verification of Snell's law) was experimentally observed by Repp et al. in the $2 \mathrm{ML} \mathrm{NaCl} / \mathrm{Cu}(111)$ system. ${ }^{10}$ This system exhibits a moiré pattern and a gapped surface band structure (i.e., similar to the $1 \mathrm{ML} \mathrm{Ag} / \mathrm{Cu}(111)$ system, but with different domain orientations and smaller effective gaps. Electron refraction in this system was observed at the lateral interface between the clean $\mathrm{Cu}(111)$ surface and the insulating $2 \mathrm{ML} \mathrm{NaCl-capped}$ $\mathrm{Cu}(111)$ surface, at electron energies below the Fermi level.

Here, we propose and theoretically demonstrate that electron refraction can also be revealed at the lateral interface between the dislocation-1 $\mathrm{ML} \mathrm{Ag} / \mathrm{Cu}(111)$ system and $\mathrm{Cu}(111)$ at electron energies below the Fermi level. Since the scattering potential of $\mathrm{Ag}$ steps is very weak ${ }^{11}$ and the system assembles into one single domain of dislocations, the experimental realization of electron refraction is made easy compared to other systems. Refraction occurs because the Constant-Energy Surfaces 
(CESs) of the two media have different wave-vectors $\mathbf{k}$ and group velocities so that the refracted beam follows a direction that conserves the parallel component of $\mathbf{k}$. In contrast to homogeneous interfaces, the presence of the dislocation lattice produces umklapp bands that result in negative-refraction at high incidence angles. At such high angles, total internal reflection takes place for the intense main-band electrons, making the identification of weak negative refraction experimentally viable. We obtain excellent agreement between $\mathbf{k}$-space and realspace simulations, calculated by Electron Plane Wave Expansion (EPWE) and Boundary Element Method (EBEM) approaches, respectively. Although isotropic CESs are considered throughout this work, such an agreement allows us to schematically illustrate that spin-splitting and negative refraction could be realized for spin-polarized and/or anisotropic energy surfaces, such as those characteristic of surface alloys with Rashba-split bands and topological insulators. We further claim a general applicability of the approach followed here, provided that electronic surface states are present and lateral interfaces are properly designed.

\section{THEORY}

The surface band structures and CESs are calculated by employing EPWE, while real-space simulations of electron refraction are obtained using the EBEM. The Schrödinger equation for electrons experiencing an effective potential $V$ corresponding to an infinitely extended 2D periodic dislocation lattice is

$$
\frac{-\hbar^{2}}{2 m_{\mathrm{eff}}}\left[\nabla^{2}+k^{2}-\frac{2 m_{\mathrm{eff}}}{\hbar^{2}} V(x, y)\right] \psi_{n \mathbf{k}}=0,
$$

where $k=\frac{\sqrt{2 E m_{\mathrm{eff}}}}{\hbar}$ is the electron wavenumber, $E$ is its energy, and $m_{\text {eff }}$ is the effective mass. Notice that the wave functions are labeled by the Bloch wave vector $\mathbf{k}$. In EPWE, we expand the wave functions $\psi_{n \mathbf{k}}$ and the potential $V$ as a sum of Fourier components running over the reciprocal lattice vectors of the periodic structure G. Inserting these expansions inside Eq. (1), we find the linear system of equations $^{12}$

$$
\left(\hbar^{2} k^{2} / 2 m_{\mathrm{eff}}-E\right) \psi_{n \mathbf{k}, \mathbf{G}}+\sum_{\mathbf{G}^{\prime}} V_{\mathbf{G}-\mathbf{G}^{\prime} \psi n \mathbf{k}, \mathbf{G}^{\prime}}=0,
$$

where $V_{\mathbf{G}}$ and $\psi_{n \mathbf{k}, \mathbf{G}}$ are the Fourier coefficients of $V$ and $\psi_{n \mathbf{k}}$. In practice we solve the system for a finite number of G's, limited by the condition $G<g_{\max } 2 \pi / a$, where $a$ is the period of the dislocation lattice. We obtain numerically converged results with $g_{\max } \sim 10$.

In the EBEM, the solution of Eq. (1) is obtained through a fine discretization scheme of the boundaries enclosing regions with different potentials. Equivalent electron sources are placed at these boundaries and propagated through each region $j$ of potential $V_{j}$ by means of the $2 \mathrm{D}$ Green function of Helmholtz equation as detailed in Ref. 13.

In both approaches, the potential modulations due to the detailed atomic structure were not considered, which is a good approximation for surface states with typical wavelengths larger than the atomic spacing.

\section{RESULTS AND DISCUSSION}

Our model system is depicted in Fig. 1(a). The blue region defines the structureless $\mathrm{Cu}(111)$ surface, where the potential $V_{o}$ is set to zero, and the red-green region is the 1 $\mathrm{ML} \mathrm{Ag} / \mathrm{Cu}(111)$ dislocation lattice with an effective potential variation $\Delta V=V_{2}-V_{1}=650 \mathrm{meV}$. ${ }^{9}$ The solid-white, solid-black, and dashed-white arrows mark the direction of the incident, refracted, and reflected electron beams at the concerned interface, respectively, for which the electron energy and incident angle are varied. In Fig. 1(b), we present the calculated band structures along the surface-projected $\overline{\Gamma M}$ directions for the $1 \mathrm{ML} \mathrm{Ag} / \mathrm{Cu}(111)$ and $\mathrm{Cu}(111)$ systems. Calculations are performed by EPWE for each system separately. An effective mass of $0.41 m_{e}$ and values of various potentials of $V_{o}=0, V_{1}=150 \mathrm{meV}$, and $V_{2}=800 \mathrm{meV}$ are used, while the reference energy is set to $400 \mathrm{meV}$ below the Fermi level (i.e., the binding energy of the $\mathrm{Cu}(111)$ surface state). The $\bar{\Gamma}$-point energy of the $1 \mathrm{ML} \mathrm{Ag} / \mathrm{Cu}(111)$ is found at $200 \mathrm{meV}$, and the $\bar{M}$-point gap size amounts to $\sim 60 \mathrm{meV}$, in agreement with previous experiments and calculations. $^{7-9,14,15}$ Here, electron refraction is investigated at two selected energies (dashed lines in (b)): $E_{B}=53 \mathrm{meV}$ and $E_{B}=160 \mathrm{meV}$. The corresponding CESs are circular with larger $\mathbf{k}$ for $\mathrm{Cu}(111)$ at the respective energy [see Fig. 1(c)]. The CESs of the $1 \mathrm{ML} \mathrm{Ag} / \mathrm{Cu}(111)$ medium, additionally, exhibit weak umklapp contours at high $\mathbf{k}$. The coexistence of the two surface states is experimentally possible at submonolayer Ag coverage. In Fig. 1(d), the experimental photoemission intensity along the $\overline{\Gamma M}$ direction for $\sim 0.6 \mathrm{ML}$ $\mathrm{Ag} / \mathrm{Cu}(111)$ is presented. Both the $\mathrm{Cu}(111)$ and the $1 \mathrm{ML}$ $\mathrm{Ag} / \mathrm{Cu}(111)$ surface states coexist with their band minima at $\sim 400 \mathrm{meV}$ and $\sim 200 \mathrm{meV}$, respectively. The overall matching with the calculations is highlighted by superimposing the dispersions in (b) onto the photoemission intensity. At such a reduced coverage, the geometry defined in Fig. 1(a) is frequently present in STM measurements [see Fig. 3 in Ref. 9]. In contrast to the $2 \mathrm{ML} \mathrm{NaCl} / \mathrm{Cu}(111)$ system, a monoatomic step height and single domain Ag stripes are obtained in flat ${ }^{9}$ or vicinal ${ }^{16} \mathrm{Cu}(111)$, making the experimental observation of electron refraction relatively simple.

In Figs. 2(a) and 2(b), we present for the geometry defined in Fig. 1(a) the wave-vector-space electron bands for the two sets of CESs shown in Fig. 1(c). The top and bottom halves are the CESs for $1 \mathrm{ML} \mathrm{Ag} / \mathrm{Cu}(111)$ and $\mathrm{Cu}(111)$, respectively. An electron beam of wave-vector $\mathbf{k}_{\text {in }}$ (blue arrow), coming from $\mathrm{Cu}(111)$ and hitting the interface at an angle $\alpha$, undergoes side refraction (red arrow) with a new wave-vector $\mathbf{k}_{\text {refr }}$ and refraction angle $\beta$ following Snell's law. In wave-vector-space, the direction of any transmitted beam (i.e., $\beta$ ) is obtained from the intersections of construction lines placed at $\mathbf{k}_{\text {in }}$ (dashed-vertical lines) with the CES at the second interface, i.e., $\mathrm{Ag} / \mathrm{Cu}(111)$. These lines set the conservation constrains for the wave-vector components parallel to the interface. Except for normal incidence, a partial reflection simultaneously occurs (dashed-blue arrow) with $\left|\mathbf{k}_{\text {refl }}\right|=\left|\mathbf{k}_{\text {in }}\right|$, and the intensity of this reflected beam is enhanced for large incidence angles according to Fresnel's coefficients. The phases are also matched at the interface and 


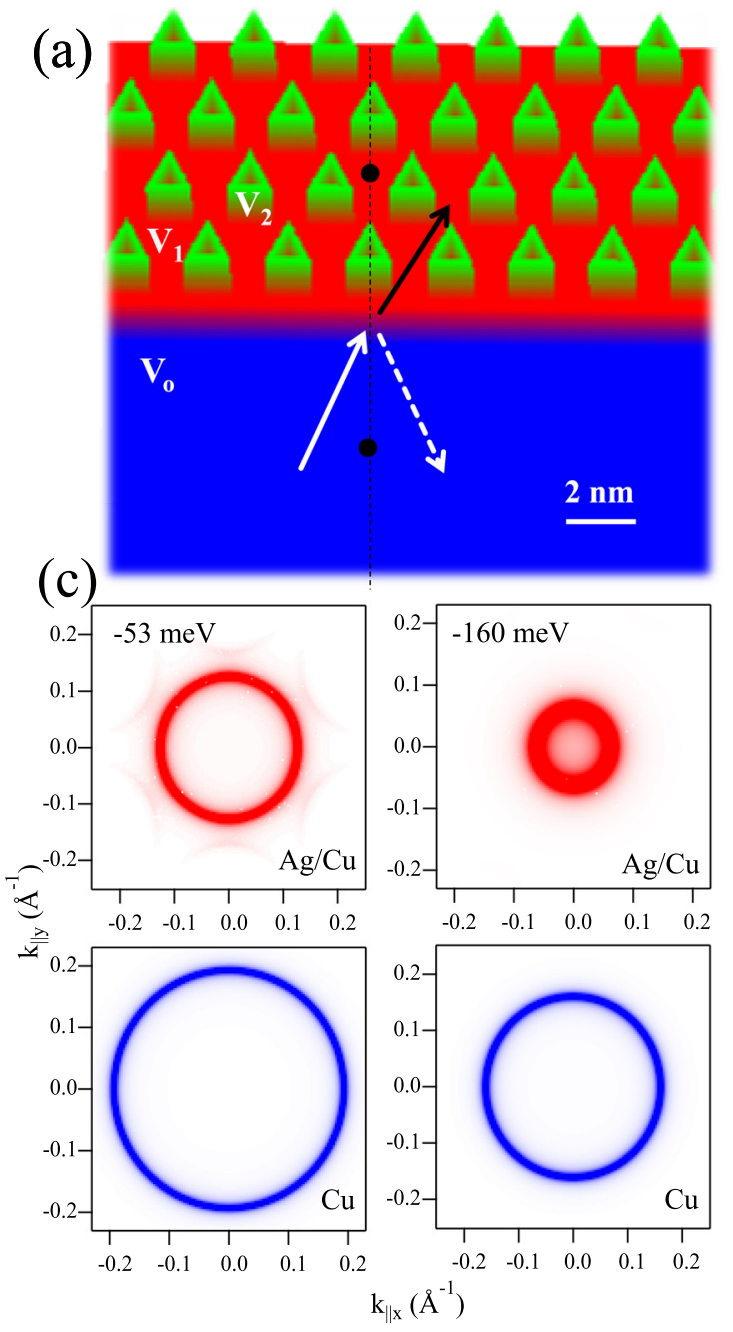

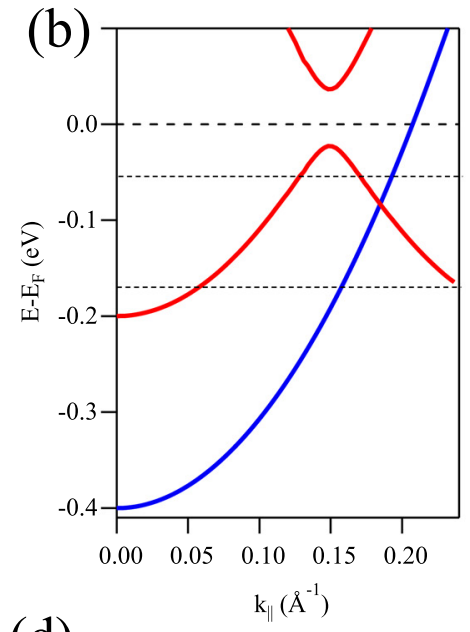

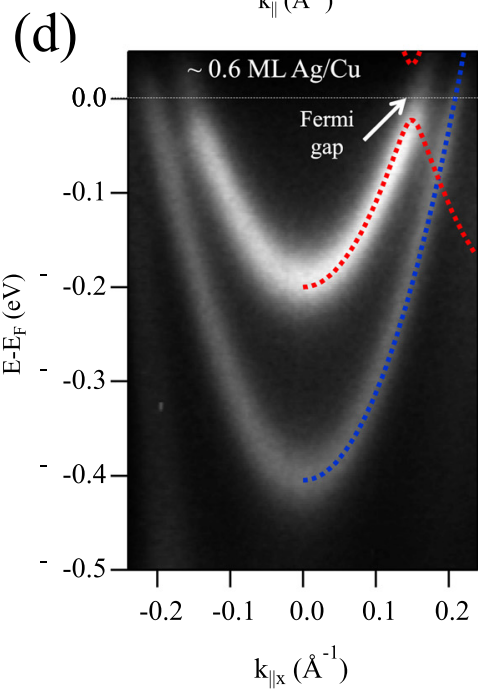

FIG. 1. (a) Geometry used in EBEM and EPWE calculations. The red and blue areas represent the moiré-1 ML $\mathrm{Ag} / \mathrm{Cu}(111)$ and the clean $\mathrm{Cu}(111)$ surface, respectively, while the green triangles describe the dislocation lattice characteristic of the annealed moiré-1 ML $\mathrm{Ag} / \mathrm{Cu}(111)$ surface. The arrows represent the incidence (solid-white), refracted (solid-black), and reflected (dashed-white) beams. (b) Calculated band structures using EPWE for the dislocation-1 ML $\mathrm{Ag} / \mathrm{Cu}(111)$ (red) and the clean $\mathrm{Cu}(111)$ (blue) surfaces along the $\overline{\Gamma M}$ direction. (c) Simulated constant energy surfaces taken at the dashed-black lines in (b) (i.e., at $-160 \mathrm{meV}$ and $-53 \mathrm{meV}$ ) for both dislocation-1 ML $\mathrm{Ag} / \mathrm{Cu}(111)$ (top) and $\mathrm{Cu}(111)$ (bottom) surfaces. (d) Experimental ARPES data taken along the $\overline{\Gamma M}$ direction for a $\sim 0.6 \mathrm{ML} \mathrm{Ag} /$ $\mathrm{Cu}(111)$. are $\pi$ out-of-phase for the refracted and reflected beams, respectively. At the critical angle $\alpha_{c}$ (black arrow), the beam is transmitted parallel to the interface (dashed-black arrow) forming an evanescent wave so that total internal reflection proceeds at higher angles. The momentum-space form of Snell's law is written as

$$
\left|k_{\text {in }}\right| \sin (\alpha)=\left|k_{\text {refr }}\right| \sin (\beta) ; \quad \lambda_{\text {refr }} \sin (\alpha)=\lambda_{\text {in }} \sin (\beta) .
$$

All these quantities are summarized in Table I for the two concerned binding energies and for selected incidence angles. Before we present a real-space representation of refraction for the finite geometry presented in Fig. 1(a) using EBEM, we want to ensure that the EPWE calculated band structures for each region are unaltered in the finite geometry. To this end, we present Local Density of States (LDOS) calculations obtained from EPWE [see Fig. 2(c)] for $\mathrm{Cu}(111)$ (blue) and 1 $\mathrm{ML} \mathrm{Ag} / \mathrm{Cu}(111)$ (red) systems. The corresponding LDOS for the finite geometry is calculated at the spatial location of two black dots indicated in Fig. 1(a). The agreement between LDOS for the extended and finite geometries is reassuring (i.e., neither the shift of the surface state energy nor the variation in the $\bar{M}$-point gap size or energetic position are observed).

Figure 3 presents EBEM simulation results of refraction-reflection behavior for a Gaussian electron beam, made up of $\sim 100$ plane waves, hitting the $\mathrm{Cu}(111)-1 \mathrm{ML}$
$\mathrm{Ag} / \mathrm{Cu}(111)$ interface at the tabulated incidence angles with a Gaussian wave-vector distribution along the direction transversal to the beam propagation. The beam width is set to $\sim 100 \AA$, with its focal point placed right at the interface. The yellow-black arrows positioned according to Eq. (3) corroborate the good matching between the real-space simulations and wave-vector-space analysis, except for critical angle incidence [see Fig. 3(e)]. Here, the transmitted intensity is not parallel to the interface (green arrow) and deviates by $\sim 9^{\circ}$ from the wave-vector-space analysis (black arrow). In fact, close to $\alpha_{c}$, the angle $\beta$ is highly sensitive to small variations in $\alpha$, where a deviation by only $1^{\circ}$ leads to a change of $\beta$ by $\sim 4^{\circ}$. Given the finite width of the beam employed here (i.e., $\sim 100 \AA$ ), different incidence angles, around the concerned one, are simultaneously sampled. ${ }^{17}$ This effect is also presented in Fig. 3(d), for which traces of transmitted intensity, for $\alpha>\alpha_{c}$, are faintly visible. In such circumstances, the direction of the refracted beam is better estimated from the phase analysis presented in Fig. 3(f), where an incident plane wave is used instead. The black lines define the wavefront, and the refraction angle is found to be $\sim 90^{\circ}$ (i.e., parallel to the interface). Additionally, the phase matching condition at the interface (i.e., the continuity of the wave fronts) is clear, and the electron wavelength, defined by the green double arrow, changes its value after crossing 

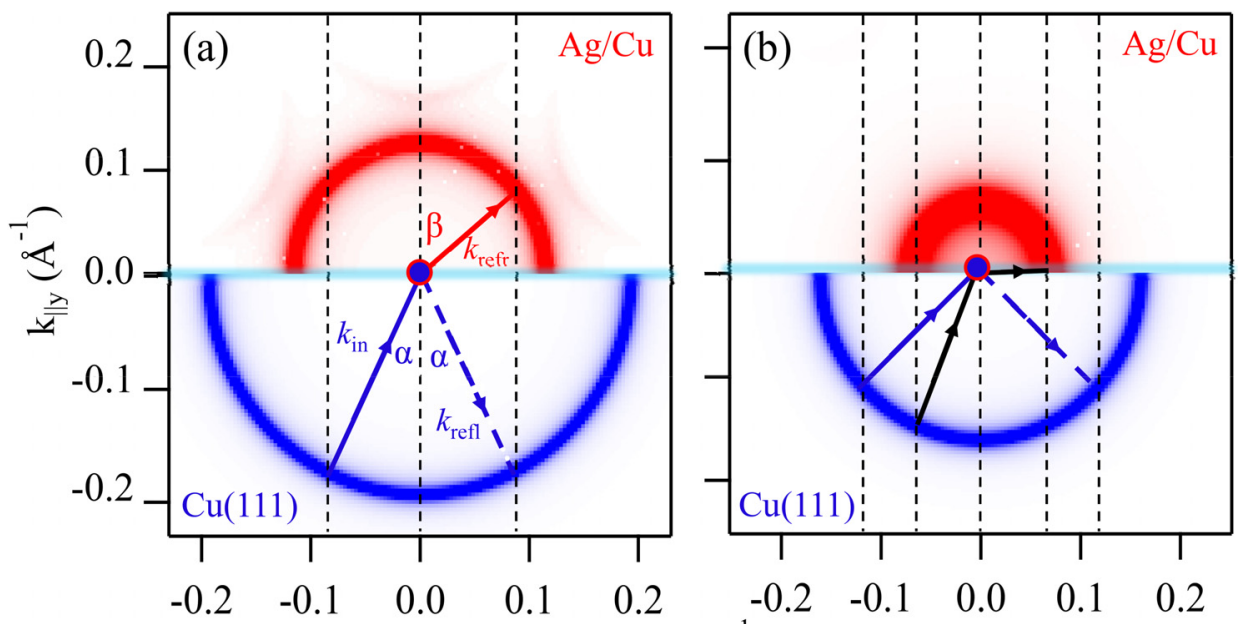

(c)

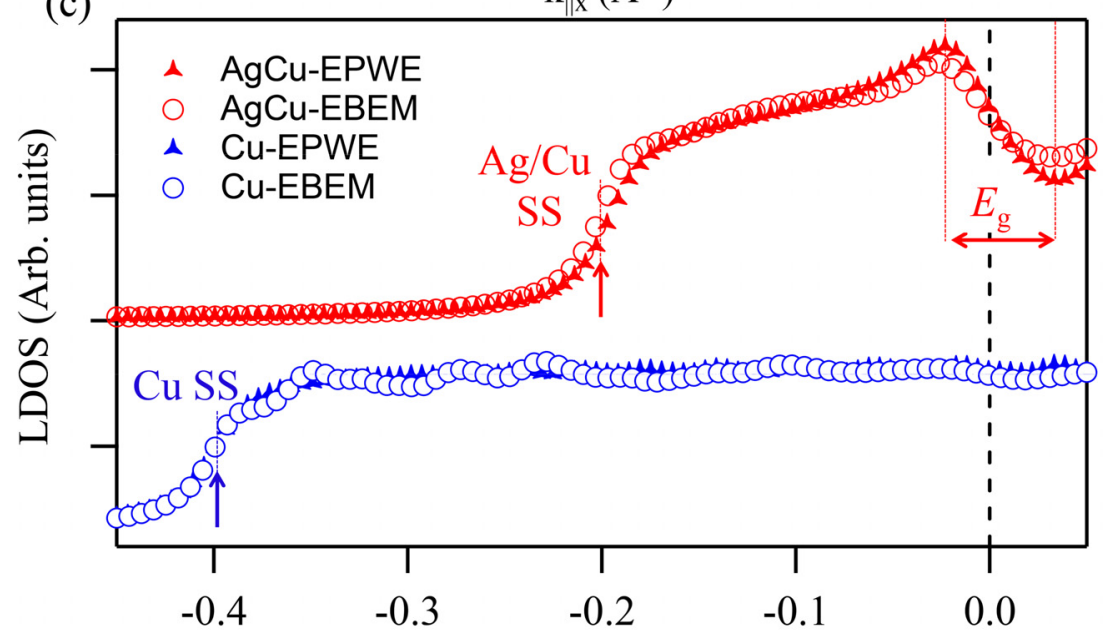

FIG. 2. Wave-vector-space analysis of the refraction/reflection at the $1 \mathrm{ML}$ $\mathrm{Ag} / \mathrm{Cu}(111)-\mathrm{Cu}(111)$ interface for electron energies (a) $E_{B}=53 \mathrm{meV}$ and (b) $E_{B}=160 \mathrm{meV}$. Upper and lower halves of (a) and (b) are the $1 \mathrm{ML} \mathrm{Ag/}$ $\mathrm{Cu}$ and $\mathrm{Cu}(111)$ constant-energy surfaces, respectively. The arrows represent electron beams with incidence angle $\alpha$ (solid-blue arrow), refracted angle $\beta$ (solid-red arrow), and reflected angle $\alpha$ (dashed-blue). The solid-black arrow represents incidence at a critical angle, and while the dashed arrow defines the direction of the confined evanescent wave. (c) Calculated LDOS at $\mathrm{Ag} / \mathrm{Cu}$ (red) and $\mathrm{Cu}(111)$ (blue) using EPWE (triangles) and EBEM (circles). In EBEM, the LDOS is calculated at the black dots in Fig. 1(a).

\section{$\mathrm{E}-\mathrm{E}_{\mathrm{F}}(\mathrm{eV})$}

the interface from $\sim 32 \AA$ to $\sim 49 \AA$, in reasonable agreement with tabulated values (Table I). Finally, the dashed black arrows in Figs. 3(a)-3(c) mark back-reflected beams at the borders of the $\mathrm{Ag} / \mathrm{Cu}$ slab.

We note that the behavior shown in Fig. 3 is practically identical, except for strong intensity modulation of the transmitted beam, to refraction/reflection at lateral interfaces between homogeneous media with different k-radii energy contours, such as submonolayer $\mathrm{Ag}$ on $\mathrm{Au}(111) .{ }^{17}$ The intrinsic fingerprint of the nanostructured dislocation lattice is shown in Fig. 4(a). At $50^{\circ}$ (top) and $60^{\circ}$ (bottom) incidence, in addition to the reflected beams (yellow dashed arrows), transmitted negatively refracted beams (black arrows) with refraction angles of $-63^{\circ}$ and $-53^{\circ}$ are also observed, respectively, which are not expected according to Eq. (3). In fact, Snell's law in the presence of a hexagonal periodic structure takes the following form ${ }^{18}$

$$
\left|\mathbf{k}_{\text {in }}\right| \sin (\alpha)+\frac{G}{2}=\left|\mathbf{k}_{\text {refr }}\right| \sin (\beta)
$$

where $G$ is a reciprocal lattice vector, which is given by either $G=\frac{4 n \pi}{a}$ or $G=\frac{4 n \pi}{\sqrt{3} a}$ if the interface is oriented along the $\overline{\Gamma K}$ or $\overline{\Gamma M}$ directions, respectively, where $n=0, \pm 1, \pm 2, \ldots$ Therefore, umklapp bands at higher-order Brillouin Zones (BZs) participate in the refraction process through the $G / 2$ term in Eq. (4). This is schematically presented in Fig. 4(b) for the $\overline{\Gamma K}$-oriented interface shown in Fig. 1(a). At such high incidence angles (see blue arrow, $k_{i n}$ ), only umklapp bands at the second BZ satisfy the parallel-momentum conservation condition (solid-red arrow, $k_{r}$ ), and the direction of the refracted beam is found by folding it back onto the first BZ (dashed-red arrow, $k_{r^{\prime}}$ ) by the appropriate reciprocal lattice vector (dashed-black arrow, $G$ ), yielding good agreement with the real-space simulation presented in (a). We note that $\mathrm{Ag} / \mathrm{Cu}$ strips are always found along the $\overline{\Gamma K}$ direction in the experiment, grown on either flat or vicinal surfaces. Nonetheless, according to Eq. (4), a different behavior is expected if the stripes were aligned along the $\overline{\Gamma M}$ direction. Closer inspection into Fig. 4(a) shows that the negatively reflected beams additionally exhibit intensities that follow the photoemission spectral weight (i.e., higher intensity at small incidence below the critical angles in accordance with the higher umklapp spectral weight near the BZ boundaries). Furthermore, the evanescent field associated with the total internal reflection within the main bands is visible at the interface, with stronger intensity suppression for $\alpha=60^{\circ}$. We note that only a totally reflected beam is obtained (not shown) at incidence angles higher than the critical angle for $E_{B}=160 \mathrm{meV}$. Obviously, the umklapp bands in this case are not met by the momentum window spanned by the $\mathrm{Cu}(111) \mathrm{CES}$ at the same energy. 

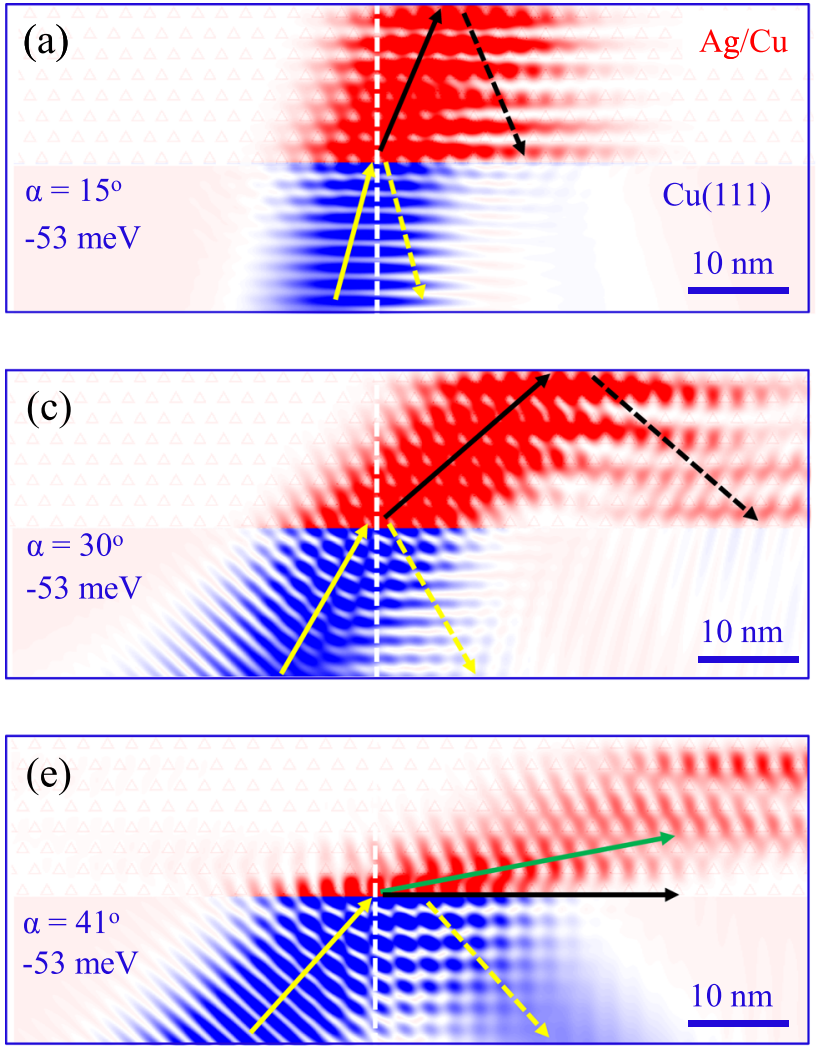
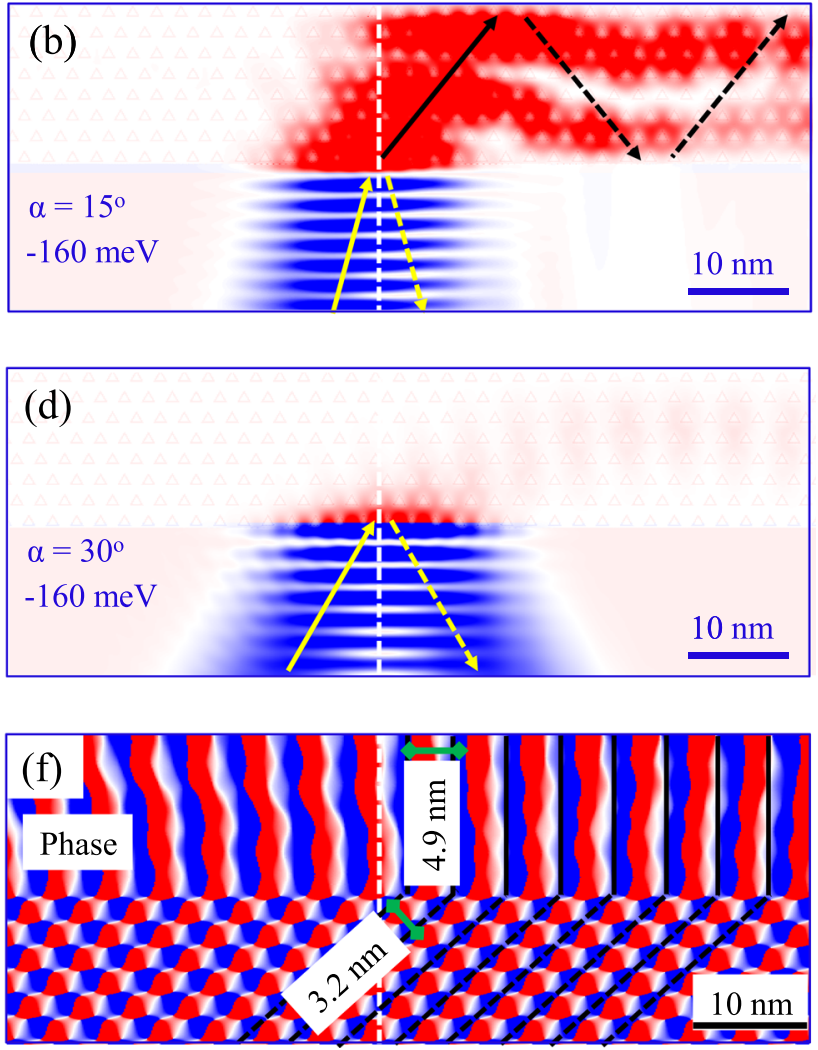

FIG. 3. Real-space simulations of electron refraction/reflection at a $\mathrm{Cu}(111)-1 \mathrm{ML} \mathrm{Ag} / \mathrm{Cu}$ lateral interface. Calculations are performed with a Gaussian electron beam incident from $\mathrm{Cu}(111)$ at angles of (a) and (b) $15^{\circ}$, for $E_{B}=53 \mathrm{meV}$ and $E_{B}=160 \mathrm{meV}$ electrons, (c) and (d) $30^{\circ}$ for $E_{B}=53 \mathrm{meV}$ and $E_{B}=160 \mathrm{meV}$ electrons, and (e) $41^{\circ}$ for the $E_{B}=53 \mathrm{meV}$ electrons only. (f) Same as (e) for a plane-wave source, only showing the corresponding phase analysis. The directions obtained from Eq. (3) and wave-vector-space analysis are defined by the solid yellow and black arrows for the incident and refracted beams, respectively, while dashed arrows stand for reflected beams from the first interface (yellow) and the second termination of the Ag/Cu slab (black). The green arrow in (e) highlights the deviation between real-space and wave-vector-space representations.

In fact, negative refraction has been recently predicted ${ }^{19}$ and experimentally verified ${ }^{20}$ for graphene $p-n$ junctions, and the fabrication of superlenses based on graphene is claimed to be possible., ${ }^{20,21}$ Although the electron-light analogy for refraction, waveguiding, collimation, etc. could be demonstrated experimentally under special conditions in some systems, the fabrication/operation of surface-state-based devices requires far more robust surface states than those reported on metal surfaces and on graphene $p-n$ junctions. In this regard, Rashba systems, such as $\mathrm{BiCu}_{2}$ or $\mathrm{BiAg}_{2}$ alloys, and topological insulators support these types of robust surface states. Moreover, the states are spin-polarized and feature highly anisotropic Fermi surfaces, ${ }^{2,23}$ making them ideal candidates for the realization of a variety of SSNE devices. In Fig. 4(c), a schematic illustration of electron refraction at the lateral interface between $\mathrm{Cu}(111)$ and $\mathrm{BiCu}_{2} / \mathrm{Cu}(111)$ is sketched. The

TABLE I. Wave vectors and wavelengths of the incidence/refracted electron beams under consideration and their critical $\left(\alpha_{c}\right)$, incidence $(\alpha)$, and refraction $(\beta)$ angles.

\begin{tabular}{lccccccc}
\hline \hline $\begin{array}{l}\mathrm{E}_{\mathrm{B}} \\
(\mathrm{meV})\end{array}$ & $\begin{array}{c}\left|\mathbf{k}_{\text {in }}\right| \\
\left(\AA^{-1}\right)\end{array}$ & $\begin{array}{c}\lambda_{\text {in }} \\
(\AA)\end{array}$ & $\begin{array}{c}\left|\mathbf{k}_{\text {refr }}\right| \\
\left(\AA^{-1}\right)\end{array}$ & $\begin{array}{c}\lambda_{\text {refr }} \\
(\AA)\end{array}$ & $\begin{array}{c}\alpha_{\mathrm{c}} \\
(\mathrm{deg} .)\end{array}$ & $\begin{array}{c}\alpha \\
(\text { deg. })\end{array}$ & $\begin{array}{c}\beta \\
(\text { deg. })\end{array}$ \\
\hline 53 & $\sim 0.19$ & 32.5 & $\sim 0.13$ & 48.9 & $\sim 41$ & 15 & $\sim 23$ \\
& & & & & & 30 & $\sim 49$ \\
160 & $\sim 0.16$ & 39.3 & $\sim 0.07$ & 94.5 & $\sim 25$ & 15 & $\sim 38$ \\
& & & & & 30 & $\cdots$ \\
\hline \hline
\end{tabular}

corresponding Fermi surfaces are represented by the black and red-blue circles for the $s p$ Shockley and $p_{z} E_{+} / E_{-}$Rashba surface states, ${ }^{24}$ respectively.

At the interface between such electron-like (i.e., phase $\left(v_{\mathrm{ph}}\right)$ and group $\left(v_{\mathrm{g}}\right)$ velocities are parallel) and hole-like (i.e., phase $\left(v_{\mathrm{ph}}\right)$ and group $\left(v_{\mathrm{g}}\right)$ velocities are anti-parallel) bands, momentum conservation implies that the tangential component of the group velocity reverses its direction, leading to the negative refraction illustrated in Fig. 4(c). Furthermore, two spin-polarized refracted electron beams (red and blue arrows) are obtained, demonstrating the possible application of such a system as a spin-filter, and providing a clear analogy to birefringent materials. We note that in contrast to the $\mathrm{Cu}-\mathrm{Ag} / \mathrm{Cu}$ interface, the fabrication of $\mathrm{Cu}-\mathrm{BiCu}_{2}$ lateral interfaces cannot be made by $\mathrm{Bi}$-submonolayer coverage, but possibly by masking a portion of $\mathrm{Cu}(111)$ single crystals during $\mathrm{Bi}$ deposition. Interestingly, unlike the $\mathrm{Cu}-\mathrm{Ag} / \mathrm{Cu}$ system, lateral interfaces with such Rashba alloys do not involve a "physical" atomic step, and therefore, electron transmission is greatly enhanced.

The negative refraction expected in this particular system is analogous to that of a subwavelength metamaterial in which the effective refractive index is negative. Negative refraction without a negative refractive index is also possible in photonic crystals with suitable shapes of their iso-energy contours. ${ }^{25}$ Electron analogues for this effect could be found at the interface between $\mathrm{Bi}_{2} \mathrm{Se}_{3}{ }^{22}$ with a circular Fermi contour and $\mathrm{Bi}_{2} \mathrm{Te}_{3}{ }^{23}$ with an anisotropic Fermi surface at certain 


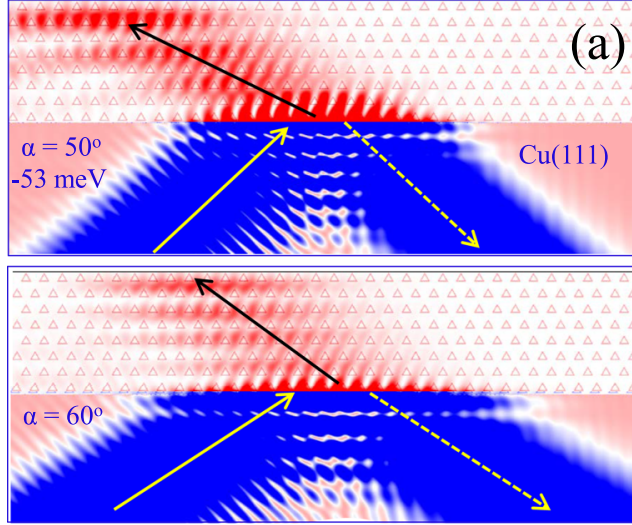

(c)

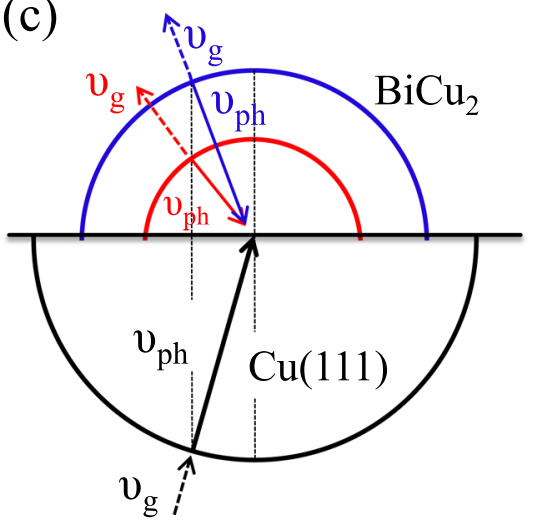

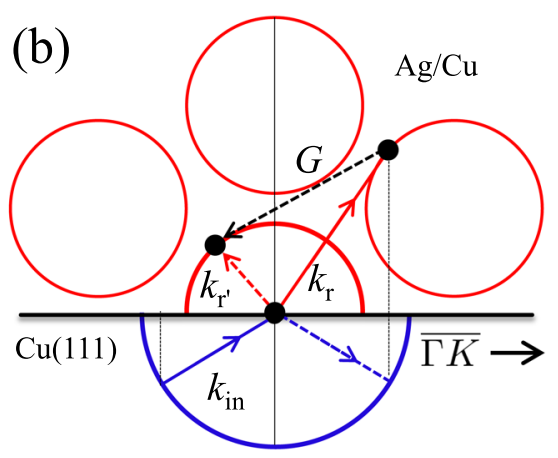

(d)

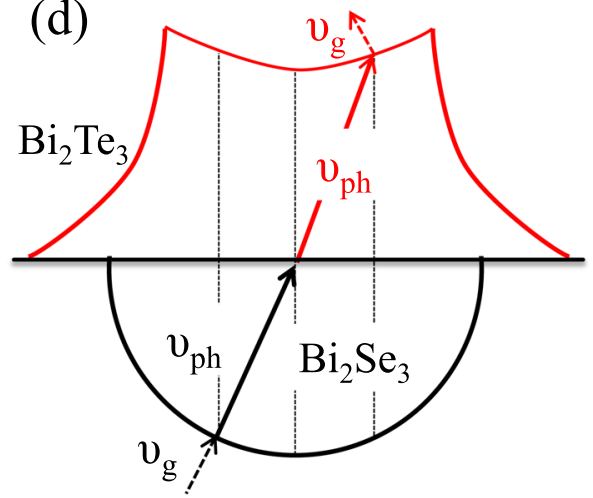

FIG. 4. (a) Real-space simulations for electron negative-refraction at the $\mathrm{Cu}(111)-1 \mathrm{ML} \mathrm{Ag} / \mathrm{Cu}$ interface, where the interface is orientated along the $\overline{\Gamma K}$ direction, for incidence angles of $50^{\circ}$ (top) and $60^{\circ}$ (bottom). (b) Wavevector-space representation of the refraction/reflection in (a). Solid blue and red arrows represent incidence and positive/umklapp reflected beams, respectively, while dashed red arrows define the direction of the negatively refracted electrons. (c) and (d) Wavevector-space diagrams for the interfaces: (c) $\mathrm{Cu}(111)-\mathrm{BiCu}_{2}$ surface alloy and (d) $\mathrm{Bi}_{2} \mathrm{Se}_{3}-\mathrm{Bi}_{2} \mathrm{Te}_{3}$. The directions of $v_{\mathrm{ph}}$ and $v_{\mathrm{g}}$ are denoted by solid and dashed arrows, respectively. Black and red/blue (i.e., for electrons with different spins) arrows define the directions of the incident and refracted beams, respectively. incidence angles, as illustrated in Fig. 4(d). Both materials display electron-like dispersions, and the direction of $v_{\mathrm{g}}$ is not reversed, but negative refraction occurs here due to the curvature of the $\mathrm{Bi}_{2} \mathrm{Te}_{3}$ Fermi surface, where $v_{\mathrm{g}}$ takes the direction defined by the tangent of the dispersion $\mathrm{d} E(k) / \mathrm{d}(\hbar k)$.

We want to stress that the negative refraction observed in our model system is a result of the umklapp bands and therefore extremely weak to be realized experimentally and integrated in practical devices. In contrast, for the systems presented in Figs. 4(c) and 4(d), the main bands are doing the job, and therefore, the effect should be experimentally detectable. ${ }^{20}$ Moreover, our model system is fully metallic, and hence, inelastic contributions from the underlying substrate would potentially screen surface effects, adding up to the strong scattering of surface electrons by defects that are commonly present even in well-cleaned surfaces. In this context, topological insulators seem to fulfill all SSNE prerequisites, ${ }^{26}$ being insulators and hosting spin-polarized robust surface states. Research in 2D and $3 \mathrm{D}$ topological insulators is rapidly progressing, and hence, a variety of featured CESs that match different analogues of optics phenomena could be explored. It is important to note that such CESs have to be tuned to the Fermi energy, where the electron lifetime reaches a maximum value. A current challenge toward integrating topological insulators into SSNE applications will likely lie in the fabrication of such lateral topological insulator interfaces, although experiments in this direction are emerging. ${ }^{27,28}$

\section{CONCLUSION}

In conclusion, we have shown that electron refraction takes place at the lateral interface between the nanostructured dislocation lattice offered by $1 \mathrm{ML} \mathrm{Ag} / \mathrm{Cu}(111)$ and a homogeneous $\mathrm{Cu}(111)$ surface. At electron-surface-state-beam incidence angle higher than the critical angle, total internal reflection occurs in combination with transmitted negatively refracted electrons, resulting from the conservation of parallel momentum taking into consideration the dislocation lattice umklapp bands. Such negatively refracted beams are not present at electron energies for which the umklapp bands are positioned in regions of momentum space not spanned by the $\mathrm{Cu}(111)$ iso-energy contour taken at the same energy. For all incidence angles and electron energies, we obtain reasonable agreement between wave-vector-space and real-space simulations obtained by EPWE and EBEM calculations, respectively. Using this type of $\mathbf{k}$-space analysis, we schematically show that spin-split negative refraction is expected at the $\mathrm{BiCu}_{2}-\mathrm{Cu}(111)$ interface, while the isotropic-anisotropic $\mathrm{Bi}_{2} \mathrm{Se}_{3}-\mathrm{Bi}_{2} \mathrm{Te}_{3}$ interface might exhibit single-beam negative refraction.

\section{ACKNOWLEDGMENTS}

Z.M.A acknowledges Iñigo Aldazabal for technical support on the usage of OBERON cluster, located at MPC/ CFM Computer Center. This work has been supported in part by the Spanish MINECO (Grant Nos. MAT2013-46593C6-4-P, MAT2016-78293-C6-6-R, MAT2014-59096-P, and SEV2015-0522), the Basque Government (Grant No. IT621-13), the Catalan CERCA Program, Fundació Privada Cellex, and AGAUR (Grant No. 2014 SGR 1400).

${ }^{1}$ R. E. Hummel, Electronic Properties of Materials, 4th ed. (Springer Science Business Media, LLC, 2011).

${ }^{2}$ S. L. Chuang, Physics of Photonic Devices, 2nd ed. (Wiley, 2012). 
${ }^{3}$ M. Campbell, D. N. Sharp, M. T. Harrison, R. G. Denning, and A. J. Turberfield, Nature 404, 53 (2000).

${ }^{4}$ F. Reinert, G. Nicolay, S. Schmidt, D. Ehm, and S. Hüfner, Phys. Rev. B 63, 115415 (2001).

${ }^{5}$ J. E. Ortega and F. J. García de Abajo, Nat. Nanotechnol. 2, 601 (2007).

${ }^{6}$ S. C. Erwin, Nat. Nanotechnol. 11, 919 (2016).

${ }^{7}$ F. Schiller, J. Cordón, D. Vyalikh, A. Rubio, and J. E. Ortega, Phys. Rev. Lett. 94, 016103 (2005).

${ }^{8}$ A. Bendounan, F. Forster, J. Ziroff, F. Schmitt, and F. Reinert, Phys. Rev. B 72, 075407 (2005).

${ }^{9}$ F. J. García de Abajo, J. Cordón, M. Corso, F. Schiller, and J. E. Ortega, Nanoscale 2, 717 (2010).

${ }^{10}$ J. Repp, G. Meyer, and K.-H. Rieder, Phys. Rev. Lett. 92, 036803 (2004).

${ }^{11}$ J. E. Ortega, M. Corso, Z. M. Abd-el-Fattah, E. A. Goiri, and F. Schiller, Phys. Rev. B 83, 085411 (2011).

${ }^{12}$ C. Kittel, Introduction to Solid State Physics, 8th ed. (John Wiley \& Sons, Inc., 2004), Chap. 7.

${ }^{13}$ F. Klappenberger, D. Krühne, W. Krenner, I. Silanes, A. Arnau, F. J. García de Abajo, S. Klyatskaya, M. Ruben, and J. V. Barth, Phys. Rev. Lett. 106, 026802 (2011).

${ }^{14}$ Z. M. Abd El-Fattah, M. Matena, M. Corso, F. J. García de Abajo, F. Schiller, and J. E. Ortega, Phys. Rev. Lett. 107, 066803 (2011).
${ }^{15}$ D. Malterre, B. Kierren, Y. Fagot-Revurat, C. Didiot, F. J. García de Abajo, F. Schiller, J. Cordón, and J. E. Ortega, New J. Phys. 13, 013026 (2011).

${ }^{16}$ F. Schiller, M. Ruiz-Osés, J. Cordón, and J. E. Ortega, Phys. Rev. Lett. 95, 066805 (2005).

${ }^{17}$ M. A. Kher-Elden, Z. M. Abd El-Fattah, O. Yassin, and M. M. El-Okr, Phys. B: Condens. Matter 524, 127 (2017).

${ }^{18}$ S. Foteinopoulou and C. M. Soukoulis, Phys. Rev. B 72, 165112 (2005).

${ }^{19}$ V. V. Cheianov, V. Fal'ko, and B. L. Altshuler, Science 315(5816), 1252 (2007).

${ }^{20}$ G.-H. Lee, G.-H. Park, and H.-J. Lee, Nat. Phys. 11, 925 (2015).

${ }^{21}$ P. Makk, Nat. Phys. 11, 894 (2015).

${ }^{22}$ C. Chen et al., Proc. Natl. Acad. Sci. 109(10), 3694 (2012).

${ }^{23}$ M. Z. Hasan and C. L. Kane, Rev. Mod. Phys. 82, 3045 (2010).

${ }^{24}$ L. Moreschini, A. Bendounan, H. Bentmann, M. Assig, K. Kern, F. Reinert, J. Henk, C. R. Ast, and M. Grioni, Phys. Rev. B 80, 035438 (2009).

${ }^{25}$ E. Cubukcu, K. Aydin, E. Ozbay, S. Foteinopoulou, and C. M. Soukoulis, Nature 423, 604 (2003).

${ }^{26}$ P. Sessi et al., Phys. Rev. B 94, 075137 (2016).

${ }^{27}$ Y. Li, J. Zhang, G. Zheng, Y. Sun, S. S. Hong, F. Xiong, S. Wang, H. R. Lee, and Y. Cui, ACS Nano 9(11), 10916 (2015).

${ }^{28}$ S. H. Kim, K.-H. Jin, B. W. Kho, B.-G. Park, F. Liu, J. S. Kim, and H. W. Yeom, ACS Nano 11, 9671 (2017). 